\title{
BMJ Open Omega-3 polyunsaturated fatty acid supplementation for improving peripheral nerve health: protocol for a systematic review
}

Alexis Ceecee Zhang, ${ }^{1}$ Richard J Maclsaac, ${ }^{2,3}$ Leslie Roberts, ${ }^{3,4}$ Jordan Kamel, ${ }^{3,4}$ Jennifer P Craig, ${ }^{5}$ Lucy Busija, ${ }^{6}$ Laura E Downie $^{1}$

To cite: Zhang AC, Maclsaac RJ, Roberts L, et al. Omega-3 polyunsaturated fatty acid supplementation for improving peripheral nerve health: protocol for a systematic review. BMJ Open 2018;8:e020804. doi:10.1136/ bmjopen-2017-020804

- Prepublication history and additional material for this paper are available online. To view these files, please visit the journal online (http://dx.doi. org/10.1136/bmjopen-2017020804).

Received 24 November 2017 Revised 20 January 2018 Accepted 21 February 2018

Check for updates

${ }^{1}$ Department of Optometry and Vision Sciences, University of Melbourne, Parkville, Victoria, Australia

${ }^{2}$ Department of Endocrinology and Diabetes, St Vincent's Hospital Melbourne, Fitzroy, Victoria, Australia

${ }^{3}$ Department of Medicine, University of Melbourne, Parkville, Victoria, Australia ${ }^{4}$ Centre for Clinical

Neurosciences and Neurological Research, St Vincent's Hospital Melbourne, Fitzroy, Victoria, Australia

${ }^{5}$ Department of Ophthalmology, New Zealand National Eye Centre, The University of Auckland, Auckland, New Zealand

${ }^{6}$ Institute for Health and Ageing, Australian Catholic University, Fitzroy, Victoria, Australia

Correspondence to

Dr. Laura E Downie;

Idownie@unimelb.edu.au

\section{ABSTRACT}

Introduction Damage to peripheral nerves occurs in a variety of health conditions. Preserving nerve integrity, to prevent progressive nerve damage, remains a clinical challenge. Omega-3 polyunsaturated fatty acids (PUFAs) are implicated in the development and maintenance of healthy nerves and may be beneficial for promoting peripheral nerve health. The aim of this systematic review is to assess the effects of oral omega-3 PUFA supplementation on peripheral nerve integrity, including both subjective and objective measures of peripheral nerve structure and/or function.

Methods and analysis A systematic review of randomised controlled trials that have evaluated the effects of omega-3 PUFA supplementation on peripheral nerve assessments will be conducted. Comprehensive electronic database searches will be performed in Ovid MEDLINE, Embase, the Cochrane Central Register of Controlled Trials (CENTRAL), US National Institutes of Health Clinical Trials Registry and the WHO International Clinical Trials Registry Platform. The title, abstract and keywords of identified articles will be assessed for eligibility by two reviewers. Full-text articles will be obtained for all studies judged as eligible or potentially eligible; these studies will be independently assessed by two reviewers to determine eligibility. Disagreements will be resolved by consensus. Risk of bias assessment will be performed using the Cochrane Collaboration risk of bias tool to appraise the quality of included studies. If clinically meaningful, and there are a sufficient number of eligible studies, a meta-analysis will be conducted and a summary of findings table will be provided.

Ethics and dissemination This is a systematic review that will involve the analysis of previously published data, and therefore ethics approval is not required. A manuscript reporting the results of this systematic review will be published in a peer-reviewed journal and may also be presented at relevant scientific conferences. PROSPERO registration number CRD42018086297.

\section{BACKGROUND}

\section{Description of the condition}

The peripheral nervous system encompasses the nerves mediating sensory, motor and
Strengths and limitations of this study

- This will be the first systematic review to consider the efficacy and safety of omega-3 polyunsaturated fatty acid (PUFA) supplementation on peripheral nerve structure and function.

- This systematic review will only consider data from randomised controlled trials, which provide the highest level of evidence for single intervention studies.

- This review will be conducted according to the Cochrane Handbook for Systematic Reviews of Interventions and in accordance with the Preferred Reporting Items for Systematic Reviews and Meta Analyses statement.

- As we will include studies that have evaluated the use of omega-3 PUFA supplementation for treating any form of peripheral nerve damage, there may be limited scope to perform a meta-analysis due to clinical heterogeneity.

- There are currently no gold standard outcome measures for assessing peripheral neuropathy, which may affect the capacity to quantitatively synthesise data from individual studies to derive clear estimates of treatment effect(s).

autonomic functions that are located outside of the brain and spinal cord. Alterations to the anatomical integrity of the peripheral nerves can adversely affect their function, presenting clinically as abnormal or loss of sensation, weakness and/or as changes to autonomic function. ${ }^{1}$ England describes peripheral neuropathy as 'a general term that indicates any disorder of the peripheral nervous system,2; this is a broad definition that includes nerve damage due to a variety of aetiologies. The pathophysiological mechanisms underlying both the development and progression of peripheral neuropathy are complex and may depend on the cause. Some of these mechanisms include altered metabolism and intracellular signalling, ${ }^{3}$ vascular 
and inflammatory stress $^{4}$ and reactive oxygen species formation. ${ }^{5}$

The most common systemic cause of peripheral neuropathy, which is evident in over $50 \%$ of individuals affected by the condition, is diabetes mellitus; the risk of peripheral neuropathy increases with longer disease duration $^{6}$ and may be correlated with the degree of glycaemic control, particularly in type 1 diabetes. ${ }^{7-10}$ Other causes include hereditary neuropathies (eg, Charcot-Marie-Tooth syndrome), post-infectious and inflammatory neuropathies (eg, Guillain-Barré syndrome) and drug-induced neuropathies (eg, platinum analogues, thalidomide and alcohol). ${ }^{11}$ Up to one-third of cases do not have an identified aetiology and are thus defined as idiopathic peripheral neuropathies. ${ }^{12}$

Clinical evaluations of peripheral nerve integrity generally include a combination of symptoms, signs and electrodiagnostic studies, which aim to evaluate the extent of nerve damage. ${ }^{2}$ Symptoms and signs of nerve damage are often assessed using validated neuropathy scales or composite scores (which combine symptomatology with clinical measures of nerve function). Nerve biopsies are invasive and, as a result, not easily repeatable and are therefore not frequently used as an outcome parameter in longitudinal studies but are instead reserved for diagnostic purposes. ${ }^{13}$

Electrodiagnostic testing examines the characteristics of the conduction of an electrical signal that travels through a single nerve. These tests are useful in providing diagnostic information and for longitudinally monitoring disease progression. ${ }^{14}$ Nerve conduction studies (NCSs) are reproducible and correlate well with underlying structural abnormalities ${ }^{15}$ but the precision of these tests is limited to detecting changes in large myelinated nerve fibres, as they are not sufficiently sensitive to detect small nerve fibre damage. ${ }^{16}{ }^{17}$ Quantitative sensory tests, which quantify thermal and pain thresholds, can be used to evaluate small nerve fibre function. ${ }^{17}$ Skin biopsies offer an alternative method to accurately diagnose and classify the extent of small fibre neuropathy, even in the absence of large fibre nerve damage. ${ }^{17}{ }^{18}$ Cutaneous silent period testing is a reproducible measurement of the nociceptive spinal reflex, where thinly myelinated A-delta fibres are the afferent arm. Quantitative sudomotor axonal reflex testing assesses the function of unmyelinated postganglionic sudomotor C fibres. ${ }^{19} 20$ These are among several other methods to assess various small fibre types, and as each individual test may have a relatively low sensitivity, a combination of modalities is usually preferable to better assess small nerve fibre function. ${ }^{21}$

Recently, corneal confocal microscopy has been applied to visualise small nerve fibres in vivo. ${ }^{22}$ This technique has been shown to correlate well with intraepidermal nerve fibre biopsy results ${ }^{23}$ and is useful for detecting and documenting various types of small fibre neuropathies. ${ }^{24-27}$ Corneal confocal microscopy has also been suggested to be useful for monitoring disease progression, and as a marker for improvements in nerve function, in the investigation of therapeutic targets for diabetic peripheral neuropathy. ${ }^{28-30}$

Peripheral neuropathies are typically treated based on their subtype and/or underlying cause(s). Treatments primarily aim to manage the underlying condition to prevent progressive nerve damage and to treat any associated symptoms. $^{21631}$ The consequences associated with symptoms of neuropathic impairment do not only affect an individual's quality of life, but also impart an economic burden in terms of healthcare costs and medical resources. ${ }^{32-34}$ This is especially true in chronic conditions, such as diabetes, where lifetime care is required. ${ }^{3}$

\section{Description of the intervention}

Omega-3 polyunsaturated fatty acids (PUFAs) are essential fatty acids (EFAs) with multiple double bonds, the first of which is located at the third carbon from the methyl end of the molecule. The short-chain omega-3 PUFA, alpha-linolenic acid, found in plant sources, is a metabolic precursor to the long-chain omega-3 PUFAs docosahexaenoic acid (DHA) and eicosapentaenoic acid (EPA), which are present in high abundance in oily fish. As humans do not have the enzymes to synthesise these fatty acids de novo, omega-3 PUFAs must be obtained from the diet or through supplementation. ${ }^{35}$ The other major class of EFAs are the omega- 6 fatty acids, which derive from the diet in the form of linolenic acid and are elongated in vivo to gamma-linoleic acid and arachidonic acid (AA). Most eicosanoids derived from the omega-6-dependent AA pathway are proinflammatory; in contrast, longchain omega-3 fatty acids bias prostaglandin metabolism towards the production of anti-inflammatory eicosanoids.

As omega-3 PUFAs competitively inhibit the metabolic conversion of omega-6 PUFAs, ${ }^{36}$ the balance of omega-3 to omega- 6 fatty acid consumption can affect systemic inflammatory processes and immune activity. The ratio of consumed omega- 6 to omega-3 in typical Western diets is approximately 15 to 1 , whereas a ratio of 4 to 1 is considered optimal. ${ }^{37}$ Increased consumption of omega-3 PUFAs is considered to provide a range of potential general health benefits, including a reduced risk of cardiovascular disease ${ }^{3839}$ and lowered systemic triglycerides. ${ }^{40}$ DHA, as an integral component in cellular membrane structures of the brain and retina, has been implicated in perinatal visual and neural development. ${ }^{41-43}$ In ocular conditions, omega-3 fatty acids supplements can reduce the symptoms and clinical signs associated with ocular surface inflammation in dry eye disease. ${ }^{44}$ The American Heart Foundation recommends a daily intake, for adults, of $500 \mathrm{mg}$ of long-chain omega-3 PUFAs ${ }^{45}$ and up to $4 \mathrm{~g}$ / day in hypertriglyceridaemia. ${ }^{46}$

\section{How the intervention might work}

Once consumed, omega-3 PUFAs alter membrane protein activity and cellular signalling response to reduce immune activity and the concentration of systemic lipid inflammatory mediators. ${ }^{47}$ The incorporation of omega-3 PUFAs into cellular membranes, and their subsequent 
effect on membrane activity, has been shown to alter vascular function, improve sciatic nerve blood flow and enhance nerve conduction velocity (NCV) in a rat model of experimental diabetic neuropathy. ${ }^{48}$

Omega-3 PUFAs also affect intracellular signalling pathways and the expression of genes, some of which may be associated with the regulation of neuron growth and neuroprotection. ${ }^{49}$ In animal models of diabetes, omega-3 PUFA supplementation has been shown to attenuate adverse changes in nerve structure and function. ${ }^{50}$ Mice enriched with genes that increase endogenous profiles of omega-3 PUFAs have been shown to have reduced neuronal cell death and increased recovery to mechanical stress and peripheral nerve injury. ${ }^{52}$ Omega-3 PUFAs have also been demonstrated to promote neurite growth in rat sensory neurons. ${ }^{53}$

Derivatives of omega-3 PUFA metabolism, resolvins and protectins, which are oxygenated metabolites from EPA and DHA, respectively, may further promote neuronal function. Neuroprotectin D-1 has been shown to facilitate the regeneration of corneal nerves following refractive surgery and neurite growth from the trigeminal ganglion of mice ${ }^{54}$ and to prevent neuropathic pain after peripheral nerve injury. ${ }^{55}$

\section{Why it is important to do this review}

This will be the first systematic review to consider the potential effects of omega-3 PUFA supplementation on peripheral nerve integrity. Omega-3 PUFA supplementation has been shown to reduce neuronal damage and enhance recovery following nerve injury in experimental animal models of peripheral neuropathy. Confirmation of these effects in clinical populations would contribute significantly towards enhancing the clinical management of peripheral neuropathy. A therapeutic agent to prevent the pathogenesis of, or slow the progression of, peripheral nerve damage has the potential to greatly improve clinical outcomes. Furthermore, the potential for omega-3 PUFA supplements to alleviate neuropathy-associated symptoms would be predicted to reduce the impairment on an individual's quality of life and lessen the economic burden of peripheral neuropathy in the community.

\section{OBJECTIVES}

The primary objective of this systematic review is to evaluate the efficacy and safety of oral omega-3 PUFA supplements for improving peripheral nerve health. Efficacy outcomes will consider both subjective endpoints (ie, symptoms) and objective clinical measures, including changes to peripheral nerve structure and function.

\section{METHODS AND ANALYSIS}

We will conduct the proposed systematic review and meta-analyses according to the recommendations stated in the Cochrane Handbook for Systematic Reviews of Interven$t_{i o n s}{ }^{56}$ and following the Preferred Reporting Items for
Systematic Reviews and Meta Analyses (PRISMA) statement. ${ }^{57}$ The protocol for this review has been registered in the PROSPERO International prospective register of systematic reviews (CRD42018086297).

\section{Eligibility criteria}

All studies published from the date of database inception until 21 November 2017 will be included. Studies will be selected according to the following eligibility criteria.

\section{Types of studies}

We will include randomised controlled trials (RCTs) where participants were allocated to consume oral omega-3 PUFA supplements. We will exclude quasi-randomised trials. We will not exclude studies based on language, publication status, year or the number of participants. In cases where more than one publication reporting data from the same cohort of participants exist (ie, from the same trial), the study reporting on the largest number of participants will be included. Published conference abstracts will be eligible for inclusion.

\section{Types of participants}

We will include studies involving adults (ie, aged 18 years or older), recruited from within any study setting, where the structure and/or function of peripheral nerves was assessed. To be eligible for inclusion in the review, studies need to include at least one subjective measure of peripheral neuropathy (eg, symptom score), one composite measure of peripheral neuropathy (ie, combining subjective and objective measures) or one objective measure of peripheral nerve structure (eg, nerve biopsy) or function (eg, NCSs).

\section{Types of interventions}

We will consider interventions where participants were randomised to oral supplementation with short-chain and/or long-chain omega-3 PUFAs. We will accept studies that administered omega-3 supplements in any form or dosage. We will exclude studies where the intervention was administered in the form of dietary manipulation (ie, a food-based intervention), and where omega-3 PUFA supplements were administered in combination with another intervention (including other nutritional interventions), unless the intervention was administered in the same dose and frequency in the comparator group. We will consider studies where omega-3 PUFA supplements were compared with placebo or no treatment.

\section{Types of outcome measures}

There are no gold standard or universally accepted outcome measures for peripheral nerve assessment. In selecting the outcome measures for this review, we considered the recommendations provided by the European Neuromuscular Centre (ENMC) International workshop: Selection of Outcome Measures for Peripheral Neuropathy Clinical Trials (10-12 December 2014), taking into account both subjective and clinical measures. ${ }^{58}$ 
We will assess all outcome measures at 6 months of follow-up, with an acceptable follow-up range of between 3 and 9 months from baseline. If studies do not report the change from baseline, we will use data reported at the end of the follow-up period.

\section{Primary outcomes}

The primary outcome will be the change, from baseline, in peripheral neuropathy impairments, as quantified by validated, composite (ie, combining symptoms plus objective measures) neuropathy measures. We have not been prescriptive in our selection of particular scales as there are no universally agreed scoring systems; examples of validated, composite neuropathy assessment scales include the Michigan Diabetic Neuropathy Score, ${ }^{59}$ Neuropathy Impairment Score (NIS) ${ }^{60}$ and Total Neuropathy Score ${ }^{61}$ For the purpose of this review, we define a 'validated' measure as a survey instrument that has been psychometrically tested.

\section{Secondary outcomes}

We will consider the following secondary outcomes:

1. Symptoms: change, from baseline, in symptoms of peripheral neuropathy, measured by a validated, patient-assessed symptom score.

2. Pain: change, from baseline, in mean scores of pain, measured by a validated, patient-assessed pain scale. Examples of validated scales include the visual analogue scale, ${ }^{62}$ Likert scales ${ }^{63} 64$ and the McGill Pain Questionnaire. ${ }^{65}$

3. Disability: change, from baseline, in the mean score of a patient-reported disability measure. Examples of validated disability measures include the Overall Neuropathy Limitation Scale ${ }^{66}$ and the Overall Disability Sum Score. ${ }^{67}$

4. Anatomical markers:

a. change, from baseline, in central corneal nerve fibre length (CNFL), defined as the total length of nerves in a given area, measured in $\mathrm{mm} / \mathrm{mm}^{2}$, using a laser-scanning in vivo confocal microscope;

b. change, from baseline, in intraepidermal nerve fibre density (IENFD).

5. NCSs: change, from baseline, in NCS parameters, as recommended by England $(2005)^{68}$ :

a. sensory nerve action potential (SNAP) amplitudes of the sural, median and ulnar nerves;

b. SNAP latencies of the sural, median and ulnar nerves;

c. sensory NCV of the sural, median and ulnar nerves;

d. distal compound motor action potential (CMAP) amplitude of the peroneal, tibial, median and ulnar nerves;

e. CMAP latency of the peroneal, tibial, median and ulnar nerves;

f. motor NCV of the peroneal, tibial, median and ulnar nerves; g. minimum F-wave latency of the peroneal, tibial, median and ulnar nerves.

6. Sensory function in the cornea: change, from baseline, in corneal sensation, as quantified using:

a. contact aesthesiometry: to quantify mechanical detection thresholds using the Cochet-Bonnet aesthesiometer (measured in millimetres);

b. non-contact aesthesiometry: to quantify corneal sensation quantified using an air-based aesthesiometer (measured in millibars).

7. Sensory function in the skin: change, from baseline, in sensory function test scores:

a. mechanical detection thresholds, measured using pressure aesthesiometry (eg, von Frey hair aesthesiometer);

b. cold detection thresholds measured using quantitative sensory testing (QST) methods;

c. warm detection thresholds measured using QST methods;

d. thermal pain thresholds, for cold and hot stimuli, measured using QST methods.

8. Adverse events: we will consider all adverse events and will analyse them in the following categories: (1) any adverse events, (2) adverse events leading to discontinuation of the interventions and (3) serious adverse events, being those leading to hospitalisation or prolonged admission, a life-threatening event or death.

\section{Search methods for identification of studies}

We will conduct comprehensive electronic database searches in: the Cochrane Central Register of Controlled Trials (CENTRAL) (Issue 10, October 2017), Ovid MEDLINE, Ovid MEDLINE Epub Ahead of Print, In-Process \& Other Non-Indexed Citations, Ovid MEDLINE Daily (January 1946-November 2017) and Embase (January 1947-November 2017). We will also search the US National Institutes of Health Clinical Trials Registry (www.clinicaltrials.gov) and the WHO International Clinical Trials Registry Platform (www.who.int/ictrp/en/). We will not impose any restrictions on date or language in our search strategies. Search strategies for all electronic searches are included in online supplementary appendices $1-5$. We will additionally search the bibliographies of included RCTs to identify any other potentially relevant studies.

\section{Data collection and analysis}

After the search strategies are performed within each electronic database, the reference lists will be imported into EndNote. Duplicate entries will be identified and removed. The final reference library will be imported into Covidence, ${ }^{69}$ the standard production platform for Cochrane systematic reviews, for the study selection process.

\section{Selection of studies}

The titles, keywords and abstracts of all unique articles, identified by the search strategies, will be independently 
reviewed by two review authors to identify potentially eligible studies. The full-text papers of articles identified as relevant or potentially relevant, by at least one reviewer, will be retrieved for full-text screening. Full-text articles will be independently screened by two review authors and assessed for eligibility according to the prespecified inclusion and exclusion criteria. Reasons for exclusion will be identified and recorded for ineligible studies that progress to the full-text screening stage. Any disagreements in eligibility assessment will be adjudicated between the two reviewers; if consensus cannot be achieved, a third independent author will be consulted to reach consensus. We will include a PRISMA flow diagram (summarising the article selection process) and a 'Characteristics of excluded studies' table (with reasons for study exclusion).

\section{Data extraction and management}

Two review authors will independently extract outcome data and key study characteristics for all included studies. Any discrepancies will be resolved by discussion and consensus. We will contact the study authors of relevant trials, by email, if further information or clarification is required. If we fail to receive a response from the corresponding author within 4 weeks, or if the authors are unable to provide us with the requested information, we will use the information that is available.

For each study, we will extract the following information:

1. article details: year of publication, journal of publication, language and publication status;

2. study details: dates study conducted, trial registration number, country, study setting, corresponding author details (name, institution, e-mail and address) and whether the study investigators were contacted for further information;

3. methods: exclusions after randomisation, losses to follow-up, how missing data were handled and whether a sample size calculation was reported;

4. participants: number of participants in each intervention group, participant baseline characteristics (ie, age, gender and/or sex (as specified by the study authors), underlying conditions, peripheral neuropathy diagnostic criteria), participant inclusion criteria and exclusion criteria and comparison of groups at baseline;

5. interventions: intervention(s) and comparator (type (long chain or short chain), dose (milligrams/day), duration of treatment), concomitant medications or treatments and compliance measures (ie, whether compliance was assessed, and the method used, eg, returned capsule counts and red blood cell fatty acid profiles);

6. outcomes: prespecified primary and secondary outcome measures and time points of assessments;

7. other: sources of funding statement (ie, present or absent), actual source of funding (eg, industry funding), conflicts of interest statement (ie, present of absent) and nature of conflict of interest (eg, industry employee).

\section{Assessment of risk of bias in included studies}

Two review authors will independently assess the risk of bias for each included study using the Cochrane Handbook for Systematic Reviews guidelines. Assessment of bias will be considered using the following domains:

1. selection bias (random sequence generation and allocation concealment);

2. performance bias (blinding of participants and all study personnel);

3. detection bias (blinding of outcome assessors);

4. attrition bias (incomplete outcome data);

5. reporting bias (selective reporting of outcomes);

6. other sources of bias (funding source and conflicts of interest).

Each review author will judge the risk of bias in each domain as: (1) low risk, (2) unclear risk or (3) high risk. Disagreements in bias assessment will be resolved by consensus between the two review authors.

\section{Measures of treatment effect}

We will analyse data according to the methods described in Chapter 9 of the Cochrane Handbook for Systematic Reviews of Interventions. ${ }^{56}$

As all of the predefined outcomes are continuous measures, we will extract information on the change in mean from baseline, and standard deviation (SD) of change, for the intervention and comparison groups. If change from baseline is not reported, we will extract information on the mean and SD of the outcome, for the intervention and comparator groups, at the specified follow-up period. The effects of the interventions will be expressed as the mean difference, with $95 \%$ confidence intervals (CIs), between the intervention and comparator groups for significant outcomes $(\mathrm{P}<0.05)$; exact $\mathrm{p}$ values will be reported.

\section{Unit of analysis issues}

The unit of analysis for this review will be the individual participant. In studies where outcomes were measured in ocular tissues, the unit of analysis will be the enrolled study eye of the participant. In studies where participants were randomly allocated to treatment, there will be no unit of analysis issues if only one eye per person is included in the trial, or if both eyes per person are included and the average value of both eyes are reported. In studies where participants were randomly allocated to treatment and both eyes were included, but reported separately, we will analyse this as clustered data (ie, adjusted for within-person correlation). We may have to contact the trial investigators for further information to do this.

\section{Dealing with missing data}

For any studies where missing outcome data (eg, omitted SD, standard errors) are identified, we will attempt to contact the study authors. If we fail to receive a response from the corresponding authors in 4 weeks, or if the authors are unable to provide us with the requested information, we will use the information that is available. We 
will use imputed data, if this has been derived by the trial investigators using an appropriate method, but will not impute missing data ourselves.

\section{Assessment of heterogeneity}

We will assess clinical and methodological heterogeneity in the included studies by examining differences in the intervention (eg, type, dose and form), participant characteristics at baseline (eg, age, gender/sex, cause of neuropathy, eligibility criteria) and risk of bias. We will quantify statistical heterogeneity using the $\mathrm{I}^{2}$ statistic, as outlined in Chapter 9 of the Cochrane Handbook for Systematic Reviews of Interventions ${ }^{56}$; we will consider an $\mathrm{I}^{2}$ statistic of $60 \%$ or more as consistent with a moderate level of heterogeneity. In measuring heterogeneity, we will also consider the: (1) magnitude and direction of the effects of individual studies and (2) strength of evidence for heterogeneity (using a $p<0.10$ from the $\chi^{2}$ test as the criterion for significant heterogeneity).

\section{Assessment of reporting biases}

If at least 10 studies are included in a meta-analysis, we will use a funnel plot to assess for any potential publication bias. We will interpret any asymmetries in the funnel plot in association with the trial characteristics, considering relevant factors such as sample size.

\section{Data synthesis}

We will undertake meta-analyses, for the primary and/ or secondary outcomes, when this would be clinically meaningful (ie, for studies where the treatment, participants and the underlying clinical questions are similar). If fewer than three RCTs are to be included in a meta-analysis, we will use a fixed-effect model; otherwise, we will use a random-effects model.

If there is inconsistency between individual study results, such that the pooled results may not provide a fair representation of the trial findings (eg, the effects are in opposite directions or $\mathrm{I}^{2}>60 \%$ or the $\chi^{2}$ test $\mathrm{p}$ value is $<0.10$ ), we will not pool the study data but will instead describe the pattern of the individual study results. If there is statistical heterogeneity but all of the effect estimates are in the same direction, such that a pooled estimate would seem to provide a good summary of the individual trial results, we may pool the data.

If a meta-analysis is not deemed appropriate, we will provide a descriptive or tabulated results summary.

\section{Subgroup analysis and investigation of heterogeneity}

If sufficient data are available, we will perform subgroup analyses by prognostic factors (eg, type of disease (including subtype of diabetes), severity of peripheral neuropathy at baseline and age) and by potential intervention effect modifiers (eg, dose, duration and type of omega-3 PUFA supplement), as these factors are potentially important to any observed treatment effects.

\section{Sensitivity analysis}

Provided there are sufficient data available, we will perform a sensitivity analysis for the primary outcome (ie, change in peripheral NIS), to assess the impact of excluding studies that: (1) were appraised as having a high risk of bias due to lack of allocation concealment or lack of blinding of participants and study personnel, (2) had more than $20 \%$ of participants that were lost to follow-up, (3) were unpublished and (4) were funded by industry.

\section{Summary of findings table}

Provided that sufficient data are available, we will provide a 'Summary of findings' table for the primary outcome (change in peripheral neuropathy as measured using a validated composite neuropathy assessment) and the following secondary outcomes (as previously defined): (1) symptoms, (2) pain, (3) CNFL, (4) IENFD, (5) SNAP amplitudes of the sural nerve and (6) motor NCV of the peroneal nerve. We will follow the recommendations specified in chapter 11 of the Cochrane Handbook for Systematic Reviews of Interventions. ${ }^{56}$ The strength and quality of the evidence for each outcome will be assessed using the Grading of Recommendations Assessment, Development and Evaluation approach. ${ }^{70}$

\section{CONCLUSIONS}

The term 'peripheral neuropathy' describes a heterogeneous group of disorders that cause damage to the peripheral nervous system. Currently, management approaches for peripheral neuropathy are aimed primarily at addressing the underlying cause and/or managing symptoms. For many causes of peripheral neuropathy, including diabetes, reversing or even limiting the progression of nerve damage remains a challenge with currently available therapeutics.

Omega-3 PUFAs are reported to be associated with a range of general health benefits that include reducing the risk of cardiovascular disease, ${ }^{38}{ }^{39}$ lowering systemic triglycerides ${ }^{40}$ and improving clinical symptoms of dry eye disease. ${ }^{44}$ In animal models of experimental peripheral nerve injury, increasing endogenous levels of omega-3 PUFAs have been shown to improve sciatic blood flow and accelerate the recovery of neuronal function. ${ }^{5052} 53$

The aim of this systematic review is to assess the safety and efficacy of oral omega-3 PUFA supplementation for improving peripheral nerve health. If it is demonstrated that omega-3 supplements can improve measures of peripheral nerve function and/or quality of life, it is anticipated that this therapy would make a valuable contribution to the current clinical management of peripheral neuropathy.

\section{Twitter@DrLauraDownie}

Acknowledgements We acknowledge the advice of Iris Gordon, Cochrane Eyes and Vision Group, who provided feedback on the search strategies for this review.

Contributors All authors made contributions to the conception and/or design of the work; ACZ and LED drafted the protocol and LB, JK, RJM, LR and JPC revised the protocol. All authors approved the final version of the manuscript and agree to be accountable for all aspects of the submitted work. 
Funding This work was supported by 2018 Melbourne Neuroscience Institute (MNI) Interdisciplinary Seed Funding (LED, RJM, LR and JK) and an Australian Government Research Training Program Scholarship (ACZ).

Disclaimer The funders had no role in the design of this protocol and will not have any role in the undertaking, data analyses, or reporting of the systematic review.

Competing interests None declared.

Patient consent Not required.

Provenance and peer review Not commissioned; externally peer reviewed. Data sharing statement Not applicable.

Open Access This is an Open Access article distributed in accordance with the Creative Commons Attribution Non Commercial (CC BY-NC 4.0) license, which permits others to distribute, remix, adapt, build upon this work non-commercially, and license their derivative works on different terms, provided the original work is properly cited and the use is non-commercial. See: http://creativecommons.org/ licenses/by-nc/4.0/

(c) Article author(s) (or their employer(s) unless otherwise stated in the text of the article) 2018. All rights reserved. No commercial use is permitted unless otherwise expressly granted.

\section{REFERENCES}

1. Hughes RA. Peripheral neuropathy. BMJ 2002;324:466-9.

2. England JD, Asbury AK. Peripheral neuropathy. Lancet 2004:363:2151-61.

3. Callaghan BC, Cheng HT, Stables CL, et al. Diabetic neuropathy: clinical manifestations and current treatments. Lancet Neurol 2012:11:521-34.

4. Cameron NE, Cotter MA. Pro-inflammatory mechanisms in diabetic neuropathy: focus on the nuclear factor kappa B pathway. Curr Drug Targets 2008;9:60-7.

5. Cashman CR, Höke A. Mechanisms of distal axonal degeneration in peripheral neuropathies. Neurosci Lett 2015;596:33-50.

6. Dyck PJ, Kratz KM, Karnes JL, et al. The prevalence by staged severity of various types of diabetic neuropathy, retinopathy, and nephropathy in a population-based cohort: the Rochester Diabetic Neuropathy Study. Neurology 1993;43:817-24

7. Nathan DM. DCCT/EDIC Research Group. The diabetes control and complications trial/epidemiology of diabetes interventions and complications study at 30 years: overview. Diabetes Care 2014;37:9-16.

8. Pop-Busui R, Boulton AJ, Feldman EL, et al. Diabetic Neuropathy: A Position Statement by the American Diabetes Association. Diabetes Care 2017:40:136-54

9. Gerstein HC, Miller ME, Byington RP, et al. Effects of intensive glucose lowering in type 2 diabetes. $N$ Engl $\mathrm{J} \mathrm{Med}$ 2008;358:2545-59.

10. Charles M, Ejskjaer N, Witte DR, et al. Prevalence of neuropathy and peripheral arterial disease and the impact of treatment in people with screen-detected type 2 diabetes: the ADDITION-Denmark study. Diabetes Care 2011;34:2244-9.

11. Martyn CN, Hughes RA. Epidemiology of peripheral neuropathy. $J$ Neurol Neurosurg Psychiatry 1997;62:310-8.

12. Gordon Smith A, Robinson Singleton J. Idiopathic neuropathy, prediabetes and the metabolic syndrome. J Neurol Sci 2006;242:9-14.

13. Sommer CL, Brandner S, Dyck PJ, et al. Peripheral Nerve Society Guideline on processing and evaluation of nerve biopsies. J Peripher Nerv Syst 2010;15:164-75.

14. Mallik A, Weir Al. Nerve conduction studies: essentials and pitfalls in practice. J Neurol Neurosurg Psychiatry 2005;76(suppl 2):ii23-31.

15. Dyck PJ, Karnes JL, Daube J, et al. Clinical and neuropathological criteria for the diagnosis and staging of diabetic polyneuropathy. Brain 1985;108(Pt 4):861-80.

16. Azhary $\mathrm{H}$, Farooq MU, Bhanushali M, et al. Peripheral neuropathy: differential diagnosis and management. Am Fam Physician 2010;81:887-92

17. Lauria G, Lombardi R. Skin biopsy: a new tool for diagnosing peripheral neuropathy. BMJ 2007;334:1159-62.

18. Hoeijmakers JG, Faber CG, Lauria G, et al. Small-fibre neuropathies-advances in diagnosis, pathophysiology and management. Nat Rev Neurol 2012;8:369-79.

19. Floeter MK. Cutaneous silent periods. Muscle Nerve 2003;28:391-401.
20. Sletten DM, Weigand SD, Low PA. Relationship of Q-sweat to quantitative sudomotor axon reflex test (QSART) volumes. Muscle Nerve 2010;41:240-6.

21. Kamel JT, Vogrin SJ, Knight-Sadler RJ, et al. Combining cutaneous silent periods with quantitative sudomotor axon reflex testing in the assessment of diabetic small fiber neuropathy. Clin Neurophysiol 2015;126:1047-53.

22. De Silva MEH, Zhang AC, Karahalios A, et al. Laser scanning in vivo confocal microscopy (IVCM) for evaluating human corneal sub-basal nerve plexus parameters: protocol for a systematic review. BMJ Open 2017;7:e018646.

23. Quattrini C, Tavakoli M, Jeziorska M, et al. Surrogate markers of small fiber damage in human diabetic neuropathy. Diabetes 2007;56:2148-54.

24. Ferdousi M, Azmi S, Petropoulos IN, et al. Corneal Confocal Microscopy Detects Small Fibre Neuropathy in Patients with Upper Gastrointestinal Cancer and Nerve Regeneration in Chemotherapy Induced Peripheral Neuropathy. PLoS One 2015;10:e0139394.

25. Asghar O, Petropoulos IN, Alam U, et al. Corneal confocal microscopy detects neuropathy in subjects with impaired glucose tolerance. Diabetes Care 2014;37:2643-6.

26. Tavakoli M, Marshall A, Thompson L, et al. Corneal confocal microscopy: a novel noninvasive means to diagnose neuropathy in patients with Fabry disease. Muscle Nerve 2009;40:976-84.

27. Sturniolo GC, Lazzarini D, Bartolo O, et al. Small fiber peripheral neuropathy in Wilson disease: an in vivo documentation by corneal confocal microscopy. Invest Ophthalmol Vis Sci 2015;56:1390-5.

28. Tavakoli M, Mitu-Pretorian M, Petropoulos IN, et al. Corneal confoca microscopy detects early nerve regeneration in diabetic neuropathy after simultaneous pancreas and kidney transplantation. Diabetes 2013;62:254-60.

29. Tavakoli M, Kallinikos P, Iqbal A, et al. Corneal confocal microscopy detects improvement in corneal nerve morphology with an improvement in risk factors for diabetic neuropathy. Diabet Med 2011;28:1261-7.

30. Pritchard N, Edwards K, Dehghani C, et al. Longitudinal assessment of neuropathy in type 1 diabetes using novel ophthalmic markers (LANDMark): study design and baseline characteristics. Diabetes Res Clin Pract 2014;104:248-56.

31. Boulton AJ, Malik RA, Arezzo JC, et al. Diabetic somatic neuropathies. Diabetes Care 2004;27:1458-86.

32. Alleman CJ, Westerhout KY, Hensen M, et al. Humanistic and economic burden of painful diabetic peripheral neuropathy in Europe: A review of the literature. Diabetes Res Clin Pract 2015;109:215-25.

33. Mehra M, Merchant S, Gupta S, et al. Diabetic peripheral neuropathy: resource utilization and burden of illness. J Med Econ 2014;17:637-45.

34. McCarberg BH, Billington $\mathrm{R}$. Consequences of neuropathic pain: quality-of-life issues and associated costs. Am J Manag Care 2006;12(9 Suppl):S263-8

35. Nettleton JA. Omega-3 fatty acids: comparison of plant and seafood sources in human nutrition. J Am Diet Assoc 1991;91:331-7.

36. Calder PC, Grimble RF. Polyunsaturated fatty acids, inflammation and immunity. Eur J Clin Nutr 2002;56(Suppl 3):S14-19.

37. Simopoulos AP. The importance of the ratio of omega-6/omega-3 essential fatty acids. Biomed Pharmacother 2002;56:365-79.

38. Simopoulos AP. The importance of the omega-6/omega-3 fatty acid ratio in cardiovascular disease and other chronic diseases. Exp Biol Med 2008:233:674-88.

39. Yokoyama M, Origasa $\mathrm{H}$, Matsuzaki M, et al. Effects of eicosapentaenoic acid on major coronary events in hypercholesterolaemic patients (JELIS): a randomised open-label, blinded endpoint analysis. Lancet 2007;369:1090-8.

40. Chauhan S, Kodali H, Noor J, et al. Role of Omega-3 Fatty Acids on Lipid Profile in Diabetic Dyslipidaemia: Single Blind, Randomised Clinical Trial. J Clin Diagn Res 2017;11:6-13.

41. Hibbeln JR, Davis JM, Steer C, et al. Maternal seafood consumption in pregnancy and neurodevelopmental outcomes in childhood (ALSPAC study): an observational cohort study. Lancet 2007;369:578-85.

42. McNamara RK. DHA deficiency and prefrontal cortex neuropathology in recurrent affective disorders. J Nutr 2010;140:864-8.

43. Birch EE, Hoffman DR, Uauy R, et al. Visual acuity and the essentiality of docosahexaenoic acid and arachidonic acid in the diet of term infants. Pediatr Res 1998;44:201-9.

44. Deinema LA, Vingrys AJ, Wong CY, et al. A Randomized, DoubleMasked, Placebo-Controlled Clinical Trial of Two Forms of Omega-3 Supplements for Treating Dry Eye Disease. Ophthalmology 2017:124:43-52. 
45. Kris-Etherton PM, Harris WS, Appel LJ, et al. Fish consumption, fish oil, omega-3 fatty acids, and cardiovascular disease. Circulation 2002;106:2747-57.

46. Miller M, Stone NJ, Ballantyne C, et al. Triglycerides and cardiovascular disease: a scientific statement from the American Heart Association. Circulation 2011;123:2292-333.

47. Gibney MJ, Hunter B. The effects of short- and long-term supplementation with fish oil on the incorporation of $n-3$ polyunsaturated fatty acids into cells of the immune system in healthy volunteers. Eur J Clin Nutr 1993;47:255-9.

48. Coste TC, Gerbi A, Vague P, et al. Neuroprotective effect of docosahexaenoic acid-enriched phospholipids in experimental diabetic neuropathy. Diabetes 2003;52:2578-85.

49. Kitajka K, Sinclair AJ, Weisinger RS, et al. Effects of dietary omega-3 polyunsaturated fatty acids on brain gene expression. Proc Natl Acad Sci U S A 2004;101:10931-6.

50. Gerbi A, Maixent JM, Ansaldi JL, et al. Fish oil supplementation prevents diabetes-induced nerve conduction velocity and neuroanatomical changes in rats. J Nutr 1999;129:207-13.

51. Yee $P$, Weymouth AE, Fletcher EL, et al. A role for omega-3 polyunsaturated fatty acid supplements in diabetic neuropathy. Invest Ophthalmol Vis Sci 2010;51:1755-64.

52. Gladman SJ, Huang W, Lim SN, et al. Improved outcome after peripheral nerve injury in mice with increased levels of endogenous $\omega-3$ polyunsaturated fatty acids. J Neurosci 2012;32:563-71.

53. Robson LG, Dyall S, Sidloff D, et al. Omega-3 polyunsaturated fatty acids increase the neurite outgrowth of rat sensory neurones throughout development and in aged animals. Neurobiol Aging 2010;31:678-87.

54. Cortina MS, He J, Russ T, et al. Neuroprotectin D1 restores corneal nerve integrity and function after damage from experimental surgery. Invest Ophthalmol Vis Sci 2013;54:4109-16.

55. Xu ZZ, Liu XJ, Berta T, et al. Neuroprotectin/protectin D1 protects against neuropathic pain in mice after nerve trauma. Ann Neurol 2013;74:490-5

56. In: Higgins J, Green S, eds. Cochrane Handbook for Systematic Reviews of Interventions Version 5.1.0. London: The Cochrane Collaboration, 2011.

57. Moher D, Liberati A, Tetzlaff J, et al. Preferred reporting items for systematic reviews and meta-analyses: the PRISMA statement. Ann Intern Med 2009;151:264-9.
58. Merkies IS, Lauria G. 131st ENMC international workshop: selection of outcome measures for peripheral neuropathy clinical trials 10-12 December 2004, Naarden, The Netherlands. Neuromuscul Disord 2006;16:149-56.

59. Feldman EL, Stevens MJ, Thomas PK, et al. A practical two-step quantitative clinical and electrophysiological assessment for the diagnosis and staging of diabetic neuropathy. Diabetes Care 1994;17:1281-9.

60. Dyck PJ, Litchy WJ, Lehman KA, et al. Variables influencing neuropathic endpoints: the Rochester Diabetic Neuropathy Study of Healthy Subjects. Neurology 1995;45:1115-21.

61. Cornblath DR, Chaudhry V, Carter K, et al. Total neuropathy score: validation and reliability study. Neurology 1999;53:1660-4.

62. Wewers ME, Lowe NK. A critical review of visual analogue scales in the measurement of clinical phenomena. Res Nurs Health 1990;13:227-36.

63. Hasson D, Arnetz B. Validation and Findings Comparing VAS vs. Likert Scales for Psychosocial Measurements. Int Electronic $\mathrm{J}$ Health Educ 2005;8:178-92.

64. Farrar JT, Young JP, LaMoreaux L, et al. Clinical importance of changes in chronic pain intensity measured on an 11-point numerical pain rating scale. Pain 2001;94:149-58.

65. Melzack R. The McGill Pain Questionnaire: major properties and scoring methods. Pain 1975;1:277-99.

66. Graham RC, Hughes RA. A modified peripheral neuropathy scale: the Overall Neuropathy Limitations Scale. J Neurol Neurosurg Psychiatry 2006;77:973-6.

67. Merkies IS, Schmitz PI, van der Meché FG, et al. Clinimetric evaluation of a new overall disability scale in immune mediated polyneuropathies. J Neurol Neurosurg Psychiatry 2002;72:596-601.

68. England JD, Gronseth GS, Franklin G, et al. Distal symmetric polyneuropathy: a definition for clinical research: report of the American Academy of Neurology, the American Association of Electrodiagnostic Medicine, and the American Academy of Physical Medicine and Rehabilitation. Neurology 2005;64:199-207.

69. Covidence systematic review software Melbourne, Australia. Veritas Health Innovation. www.covidence.org (cited 2017 November).

70. Puhan MA, Schünemann HJ, Murad MH, et al. A GRADE Working Group approach for rating the quality of treatment effect estimates from network meta-analysis. BMJ 2014;349:g5630. 